\title{
THE TYPE SET OF A TORSION-FREE ABELIAN GROUP OF RANK TWO
}

\author{
DAVID R. JACKETT
}

(Received 28 September 1978)

Communicated by $\mathbf{R}$. Lidl

\begin{abstract}
In this paper we generalize a recent result of Freedman (1973) concerning the cardinality of the type set of a rank two torsion-free abelian group. We show that if $A$ is such a group and $A$ supports a non-trivial associative ring then the type set of $A$ contains at most three elements.
\end{abstract}

Subject classification (Amer. Math. Soc. (MOS) 1970): $20 \mathrm{~K} 15$.

Throughout, the groups that we consider are abelian groups and the rings are associative rings. A ring on a group $A$ is a ring whose additive group is (isomorphic to) $A$. We write $(A, \cdot)$ for a ring on $A$ and say that $A$ supports $(A, \cdot)$. A group is called non-nil if it supports a non-trivial ring. The type set of the torsion-free group $A$ is denoted by $\mathscr{T}(A)$, and the type of $a \in A$ by $t(a)$. For the subset $S$ of the torsion-free group $A,\langle S\rangle_{*}$ denotes the unique minimal pure subgroup of $A$ containing $S$.

THEOREM 1. (Freedman (1973)). Let $A$ be a torsion-free group of rank two. If $A$ supports a ring with identity then $\mathscr{T}(A)$ contains at most three elements.

A partial generalization is contained in

THEOREM 2. (Feigelstock (1976)). Suppose $A$ is a torsion-free group of rank two, all of whose non-zero elements have non-idempotent type. Then either $A$ is nil or $|\mathscr{T}(A)|=2$.

This paper formed part of the author's Ph.D. thesis, University of Tasmania, 1977, which was written under the direction of Dr. B. J. Gardner. 
The major part of Freedman's proof consists of showing that for a torsion-free group $A$ of rank two, $\mathscr{T}(A)$ contains at most two maximal elements. More generally we can prove

Proposition 3. Let $A$ be a torsion-free group of rank $n$ with the property that every pure subgroup of $A$ of rank greater than one is non-nil. Then $\mathscr{T}(A)$ contains at most $n$ maximal elements.

PROOF. We use an induction argument. Clearly the proposition is true for a rational group; so assume that every non-nil group of rank $k(k<n)$ satisfying the conditions of the proposition has the property that its type set contains at most $k$ maximal elements. Suppose $A$ is as stated in the proposition, and let $a_{1}, a_{2}, \ldots, a_{n+1}$ be $n+1$ distinct elements of $A$ such that $t\left(a_{i}\right) \neq t\left(a_{j}\right)$ for $i \neq j$, and $t\left(a_{i}\right)$ is maximal in $\mathscr{T}(A)$ for each $i=1,2, \ldots, n+1$.

First we show that any subset of $n$ distinct elements from $\left\{a_{1}, a_{2}, \ldots, a_{n+1}\right\}$ is a maximal independent set of elements of $A$. Clearly this amounts to showing that $\left\{a_{1}, a_{2}, \ldots, a_{n}\right\}$ is an independent set of elements of $A$. If $\left\{a_{1}, a_{2}, \ldots, a_{n}\right\}$ is not independent then there exists a $k \leqslant n$ for which $\left\{a_{1}, a_{2}, \ldots, a_{k-1}\right\}$ is independent but $\left\{a_{1}, a_{2}, \ldots, a_{k}\right\}$ is not. If $A_{1}=\left\langle\oplus_{i=1}^{k-1}\left\langle a_{i}\right\rangle\right\rangle_{*}$ then $a_{k} \in A_{1}$ and, since $A_{1}$ is pure in $A$, $\mathscr{T}\left(A_{1}\right) \subseteq \mathscr{T}(A)$. But then $A_{1}$ is a rank $(k-1)$ torsion-free group satisfying the conditions of the proposition for which $\mathscr{T}\left(A_{1}\right)$ contains the $k$ maximal elements $t\left(a_{1}\right), t\left(a_{2}\right), \ldots, t\left(a_{k}\right)$. Consequently $\left\{a_{1}, a_{2}, \ldots, a_{n}\right\}$ is a maximal independent set of elements of $A$.

We can now choose a non-zero integer $m$, and integers $m_{1}, m_{2}, \ldots, m_{n}$ such that

$$
m a_{n+1}=m_{1} a_{1}+m_{2} a_{2}+\ldots+m_{n} a_{n} .
$$

If $i \in\{1,2, \ldots, n\}$ then the set $\left\{a_{1}, a_{2}, \ldots, a_{n+1}\right\} \backslash\left\{a_{i}\right\}$ is independent and so $m_{i} \neq 0$.

Consider now any ring $(A, \cdot)$ on $A$. For distinct $i$ and $j$ in $\{1,2, \ldots, n+1\}$, the maximality of $t\left(a_{i}\right)$ and $t\left(a_{j}\right)$ in $\mathscr{T}(A)$ shows $a_{i} \cdot a_{j}=0$. In particular for any $i \in\{1,2, \ldots, n\}$

$$
0=m\left(a_{n+1} \cdot a_{i}\right)=m_{i} a_{i}^{2} .
$$

Thus $m_{i} \neq 0$ yields $a_{i}^{2}=0$. Hence $(A, \cdot)$ must be the trivial ring on $A$. Since $A$ is non-nil it now follows that $\mathscr{T}(A)$ contains at most $n$ maximal elements.

Following Beaumont and Wisner (1959) we make the following definitions for the torsion-free group $A$ of rank two. If $a \neq 0$ is an element of $A$ then let

$$
Q_{a}^{\prime}=\{\alpha \in Q \mid \alpha a \in A\},
$$

where $Q$ is the group of rational numbers. Now define the nucleus $D$ of $A$ to be the subgroup $D=\bigcap_{a \in A} Q_{a}^{\prime}$ of $Q$. 
With the aid of Beaumont and Wisner (1959) the major result of Freedman (1973) can now be generalized.

THEOREM 4. Suppose $A$ is a torsion-free group of rank two that supports a nontrivial ring $(A, \cdot)$. Then $\mathscr{T}(A)$ contains at most three elements.

ProOF. We consider two cases separately.

Case (i). $(A, \cdot)$ is non-commutative. Theorem 2 of Beaumont and Wisner (1959) now gives the structure of $(A, \cdot)$; suppose $a_{1} \cdot a_{2}=\phi\left(a_{1}\right) a_{2}$ for all $a_{1}, a_{2}$ in $A$, where $0 \neq \phi \in \operatorname{Hom}(A, D)$. It is clear that $D=\left\langle p^{-\infty} \mid p A=A\right\rangle$ and also that $\operatorname{Im} \phi$ is a rank one torsion-free group with the same type as $D$. Thus $\operatorname{Im} \phi \cong D$. Hence there is a non-zero $\theta \in \operatorname{Hom}(A, D)$ such that $\theta$ maps $A$ onto $D$. We can now define a non-commutative ring $(A, \times)$ on $A$ by letting $a_{1} \times a_{2}=\theta\left(a_{1}\right) a_{2}$ for all $a_{1}, a_{2}$ in $A$. Since $1 \in D$ there is an element $a \in A$ for which $\theta(a)=1$. But then the element, $a$, will be a left identity of $(A, \times)$ and so for every $a^{\prime} \in A, t(a) \leqslant t\left(a^{\prime}\right)$. (Notice that if $(A, \cdot)$ has the alternate description in Theorem 2 of Beaumont and Wisner (1959) then we can argue as above to again obtain $t(a) \leqslant t\left(a^{\prime}\right)$.)

Case (ii). $(A, \cdot)$ is commutative. It is readily checked that $(A, \cdot)$ non-trivial and commutative implies the existence of an element $a \in A$ such that $a^{2} \neq 0$. Thus Lemma 1 of Beaumont and Wisner (1959) shows that we can choose an element $a_{1} \in A$ such that $a_{1}$ and $a_{1}^{2}$ are independent. If $a_{2}$ is a non-zero element of $A$ then there are integers $m \neq 0, m_{1}$ and $m_{2}$ such that $m a_{2}=m_{1} a_{1}+m_{2} a_{1}^{2}$. Consequently,

$$
t\left(a_{1}\right)=t\left(a_{1}\right) \cap t\left(a_{1}^{2}\right) \leqslant t\left(a_{2}\right) .
$$

In either case $\mathscr{T}(A)$ contains a smallest element. We now argue as in Freedman (1973). Since $A$ has rank two, each chain in $\mathscr{T}(A)$ is of length at most two. Proposition 3 shows $\mathscr{T}(A)$ contains at most two maximal elements. Therefore $|\mathscr{T}(A)| \leqslant 3$.

A consequence of the proof of Case (i) above is the following observation.

Proposition 5. Suppose $(A, \cdot)$ is a non-commutative ring on a torsion-free group $A$ of rank two. Then $A$ is completely decomposable.

Proof. It is clear that $D$ can be made into a rank one module over itself, that is $D$ is a projective $D$-module. As in the proof of Theorem 4 there is a non-zero $\theta \in \operatorname{Hom}(A, D)$ such that $\theta$ maps $A$ onto $D$. It is readily checked that $A$ is a $D$-module and $\theta \in \operatorname{Hom}_{D}(A, D)$. Consequently, $A$ will contain a $D$-direct summand isomorphic to $D$. Thus $A$ is completely decomposable. 


\section{References}

R. A. Beaumont and R. J. Wisner (1959), 'Rings with additive group which is a torsion-free group of rank two', Acta Sci. Math. (Szeged) 20, 105-116.

S. Feigelstock (1976), 'On the type set of groups and nilpotence', Comment. Math. Univ. St. Paul 25, 159-165.

H. Freedman (1973), 'On the additive group of a torsion-free ring of rank two', Publ. Math. Debrecen 20, 85-87.

L. Fuchs (1973), Infinite abelian groups, Vol. II (Academic Press, New York).

University of Tasmania

Hobart

Australia 